\title{
Limites y dilemas de la política económica en un país en guerra: el caso de El Salvador ${ }^{\star}$
}

\author{
Alexander Segovia
}

\section{Introducclón}

En un pais como El Salvador, que atraviesa una grave crisis económica, que padece un prolongado conflicto armado y que entrenta niveles de polarización social extremos, los dilemas, los limites y las contradicciones que se presenlan a la hora de hacer política económica son mucho mayores que los que tradicionalmente se presentan en otros países del área.

En un contexto tal, la política económica, entendida como "ese conjunto de intervenciones gubernamentales sobre las condiciones y relaciones económicas de agentes privados y públicos"1 se convierle en un campo privilegiado de lucha y generalmente en un campo más de confrontación enlre las diferentes fuerzas políticas, económicas y sociales, que la mayoría de las veces dificulta y oscurece la discusión y la solución de los problemas estriclamente económicos.

Por si eso fuera poco, la complejidad y novedad de algunos de los principales problemas económicos del pais, hace que los enfoques teóricos tradicionales, así como las recomendaciones de política que de ellos se derivan pierdan mucha de su validez. Esta situación, sumada al poco conocimiento de las transtormaciones que han tenido lugar en los últimos ańos en la estructura económica, hacen que el manejo y diseño de la política económica, en el caso de El Salvador sea una tarea sumamenle compleja y dificil.

Finalmente, hay que mencionar dos elementos que inciden de una manera decisiva en la forma de hacer política económica en EI Salvador; nos referimos a la existencia del conflicto armado y a la excesiva dependencia de la economia de la ayuda externa. 
Es denlro de la perspectiva anterior, y con el objeto de generar una discusión que posibilile encontrar vias de solución a la grave crisis económica que vive el país, a conlinuación se presentan algunas hipótesis de trabajo relacionadas con los limites, los dilemas y las contradicciones que enfrenta hoy la polílica económica en El Salvador, las cuales deberán ser desarrolladas en trabajos posteriores.

Las hipótesis a las que se hará referencia en el presente trabajo son:

1. El confliclo armado, en lanto condiciona lodos los aspectos de la vida económica del país, se convierte en el principal limite que enfrenta la política económica en El Salvador.

1.1. El impacto del conflicto armado sobre las tinanzas públicas va más allá de un simple problema contable de ingresos-gaslos. Su efeclo principal consiste en que su existencia imposibilita al gobierno cumplir de manera salisfactoria con sus funciones más elementales.

1.2. Debido a la rigidez que presentan actualmente lanto los ingresos como los gaslos gubernamentales, las posibilidades de reducir sustancialmenle el déficit fiscal más allá de los niveles actuales, es praclicamente imposible.

1.3. En el caso de El Salvador, la influencia del confliclo armado sobre la inflación no se realiza principalmente vía déficit fiscal, sino a Iravés de la presión permanente que aquel pone sobre la oferla, la demanda y las cadenas especulativas que genera.

1.4. Debido a la influencia permanente que tiene el conflicto sobre la inflación, esla tiene un limile más alla del cual no se puede avanzar mientras aquel continúe.

2. Un factor explicativo del poco éxito alcanzado en el manejo de la política económica en El Salvador en los úllimos años, es la falta de un consenso mínimo alrededor de un proyecto económico, y la falta de afinidad política e ideológica de algunos sectores con el actual gobierno.

3. Debido a lo anlerior, la politica economica, lejos de cumplir con el papel de persuación que teóricamente le corresponde, se ha convertido en un elemenlo más de confrontación entre los diferentes agentes sociales del pais.

4. En términos macroeconómicos, el sector externo es el principal límite a cualquier esluerzo de reactivación económica y es el principal punto crítico en el manejo de la política económica.

4.1. En términos de política económica exisle una contradicción entre la necesidad de mantener un nivel mínimo de estabilización, y la 
necesidad urgente de un programa de ajuste extemo.

4.2. Esta contradicción deriva del hecho de que las medidas tradicionales para hacer frente al desequilibrio externo -como la devaluación-, actúan en contra de los esluerzos de estabilización. En el caso de El Salvador, esta situación es evidenle dado el enorme impacto que tiene una medida de este tipo en los costos de producción y en el nivel de expectativas.

5. Los niveles de ayuda externa en El Salvador han llegado a un nivel tal, que ha dejado de ser un elemento complementario, para convertirse en parte constitutiva del funcionamiento de la economía salvadorefia. En este sentido, una reducción significaliva de la misma puede provocar cambios fundamentales en la esturctura económica e incluso en el modelo político del país.

6. Actualmente, el poco conocimiento que se tiene de las transformaciones que han tenido lugar en la estructura del pais, es otro de los obstáculos principales que enfrenta la polílica económica.

\section{Los limites y dilemas de la politica económica en El Salvador}

\section{II.1. El conflicto armado como el principal limite de la politica econo- mlca en El Salvador}

Generalmente, cuando se habla del impacto que tiene el conflicto armado sobre la economía del país, y especificamente de su influencia en la política económica, se hace referencia básicamente a la presión que éste pone sobre los gastos del gobierno, y por lanto sobre el déficit fiscal, el cual, bajo este enfoque, es.considerado el principal causante de la inflación. En este sentido, se argumenta que la existencia del conflicto no debería ser un obstáculo para iniciar esfuerzos serios encaminados a reactivar la economia del país. Dicho de otro modo, se considera el conflicto como una variable exógena que tiene alguna incidencia en la economía y en la política económica, pero que dicha incidencia no es de ninguna manera la más importante.

A nuestro entender, esta interpretación aparte de ser equivocada, oscurece la discusión y conlleva a enormes peligros en términos del manejo de la política económica. Como veremos a continuación, la influencia de la guerra sobre la economía de El Salvador, y sobre el manejo de la política económica va mucho más allá de su impacto en el déficit fiscal. En realidad, y ésta es nuestra tésis central, el conilicto armado en El Salvador condiciona loda la evolución económica y es el principal límile al manejo de la política económica. ${ }^{2}$ 


\section{II.1.1. La relación entre conflicto armado y déflcit flscal}

Sin duda, una de las consecuencias del conflicto armado es su impaclo sobre las finanzas públicas. Tal y como puede observarse en el cuadro No. 1, la estructura institucional del gasto gubernamental ha cambiado sustancialmente durante los últimos anos. Los gaslos por conceplo de Defensa y Seguridad Pública sumaron 7.5 por ciento del lotal de los gastos gubernamentales en 1977. A partir de ese ano, y como producto del conflicto subieron a un ritmo acelerado hasta alcanzar niveles superiores al 25 por ciento en el periodo 1985-1987, reduciendo de esla manera el presupuesto disponible para gastos sociales, servicios y otras actividades del gobierno.

Cuadro No. 1

EI Salvador. Estructura Instltuclonal de gastos gubernamentales (En porcentajes)

\begin{tabular}{|c|c|c|c|c|c|c|c|c|c|c|c|}
\hline & 1977 & 1978 & 1979 & 1980 & 1981 & 1982 & 1983 & 1984 & 1995 & 1986 & 19871 \\
\hline $\begin{array}{l}\text { 1. Denlensa y Se- } \\
\text { guridad Pública } \\
\text { 2. Admón. de deuda }\end{array}$ & 7.5 & 9.2 & 14.4 & 15.2 & 16.7 & $\begin{array}{r}18.6 \\
4\end{array}$ & 21.4 & 24.2 & 30.7 & 25.7 & 25.87 \\
\hline & 3.8 & 4.1 & 4.8 & 5.1 & 10.0 & 15.6 & 14.6 & 26.6 & 13.3 & 19.8 & 17.8 \\
\hline Sub total & 11.3 & 13.3 & 19.2 & 20.3 & 26.7 & 34.0 & 36.0 & 50.8 & 44.0 & 45.5 & 48. \\
\hline 3. Educación & 22.4 & 20.7 & 22.7 & 23.6 & 19.6 & 18.8 & 17.8 & 13.7 & 17.5 & 13.2 & 14.5 \\
\hline 4. Obras Públicas & 16.9 & 16.1 & 14.8 & 42.1 & 11.7 & 8.9 & 11.2 & 7.3 & 8.0 & 7.0 & 8.3 \\
\hline 5. Salud Pública & 10.2 & 10.7 & 9.8 & 10.5 & 9.1 & 8.2 & 9.2 & 7.0 & 7.5 & 6.2 & 8.3 \\
\hline $\begin{array}{l}\text { 6. Agria } \\
\text { Gana }\end{array}$ & 8.4 & 8.2 & 7.7 & 8.2 & 8.2 & 8.4 & 8.5 & 6.7 & 6.7 & 9.7 & 5.1 \\
\hline $\begin{array}{l}\text { 7. Translerencias } \\
\text { Generales }\end{array}$ & 2.6 & 7.9 & 3.8 & 2.6 & 2.9 & 2.9 & 2.9 & 2.4 & 2.9 & 2.0 & 7.3 \\
\hline 8. Otros & 28.2 & 23.1 & 22.7 & 22.7 & $21 . \theta$ & 14.6 & 14.6 & 12.1 & 13.0 & 16.4 & 12.9 \\
\hline al & 0.0 & 100.0 & 100.0 & 100.0 & 100.0 & 100.0 & 100.0 & 100.0 & 100.0 & 100.0 & 100.0 \\
\hline
\end{tabular}

1. Cilras Preliminares

Fuente: Minislerio de Hacienda.

Banco Central de Reserva, Sección de Finanzas Públicas.

Pese a que los datos anteriores reflejan de manera general la influencia del confliclo en el gasto gubernamental, es necesario prolundizar más en esta dirección, a tin de determinar cual es la verdadera magnitud del impacto y cómo ésle condiciona el margen de acción de la política económica en el área fiscal.

Para ello, es necesario recurrir al cuadro No. 2, que presenta la evolución y composición de los gastos de funcionamiento del Gobierno Central. Como puede verse, el rubro de Defensa y Seguridad Pública ha 
incrementado sustancialmente su participación en los gastos de funcionamiento en los últimos anos, al pasar de representar un 19.1 por ciento del total de dichos gastos en 1979 a cerca de un 50 por cienlo en el perlodo 1984-1987. Esta situación ha ido en detrimenlo del resto de unidades primarias, colocando a éstas últimas en una situación lan precaria que avanzar más en esa linea resulta prácticamente imposible. (Ver nuevamente cuadro No. 2).

Dicho en olras palabras, actualmente los gastos de funcionamiento del gobierno no relacionados con la guerra han llegado a un limite más allá del cual, cualquier recorte adicional pone en peligro el funciona-

Cuadro No. 2

El Salvador: gastos de funclonamlento del goblerno central

\begin{tabular}{c|c|c|c|c|c|c}
\hline Ahos & $\begin{array}{c}\text { Delensay } \\
\text { seguridad }\end{array}$ & $\begin{array}{c}\text { Educación } \\
\text { cultura }\end{array}$ & $\begin{array}{c}\text { Salud } \\
\text { Pública }\end{array}$ & $\begin{array}{c}\text { Obras } \\
\text { Públicas }\end{array}$ & $\begin{array}{c}\text { Otras } \\
\text { primarias }\end{array}$ & $\begin{array}{c}\text { Total } \\
\text { créditos } \\
\text { utilizados }\end{array}$ \\
\hline 1975 & 48.3 & 112.1 & 35.9 & 25.4 & 103.2 & 324.9 \\
1979 & 128.7 & 228.6 & 63.7 & 58.9 & 192.2 & 672.1 \\
1980 & 189.1 & 299.9 & 75.8 & 70.1 & 218.1 & 853.0 \\
1981 & 265.0 & 312.4 & 77.8 & 69.2 & 235.6 & 960.0 \\
1982 & 339.0 & 298.4 & 77.5 & 65.6 & 237.9 & 1.018 .4 \\
1983 & 383.0 & 284.3 & 71.0 & 59.2 & 228.8 & 1.026 .3 \\
1984 & 590.0 & 323.5 & 76.6 & 67.8 & 251.6 & 1.309 .5 \\
1985 & 710.0 & 364.1 & 77.5 & 81.4 & 259.5 & 1.492 .9 \\
1986 & 921.5 & 474.7 & 107.4 & 104.2 & 339.1 & 1.946 .9 \\
1987 & 950.9 & 504.7 & 114.6 & 120.9 & 401.3 & $2.092 .4 *$ \\
1988 & 977.9 & 536.1 & 119.2 & 124.5 & 379.9 & $2.137 .6 *$ \\
\hline
\end{tabular}

Eetructura porcentual

\begin{tabular}{l|l|r|r|r|r|l}
\hline 1975 & 14.9 & 34.5 & 11.0 & 7.8 & 31.8 & 100.0 \\
1979 & 19.1 & 34.0 & 9.5 & 8.8 & 28.6 & 100.0 \\
1980 & 22.2 & 35.1 & 8.9 & 8.2 & 25.6 & 100.0 \\
1981 & 27.6 & 32.5 & 6.1 & 7.2 & 24.6 & 100.0 \\
1982 & 33.3 & 29.3 & 7.6 & 6.4 & 23.4 & 100.0 \\
1983 & 37.3 & 27.7 & 6.9 & 5.8 & 22.3 & 100.0 \\
1984 & 45.1 & 24.7 & 5.8 & 5.2 & 19.2 & 100.0 \\
1985 & 47.6 & 24.4 & 5.2 & 5.4 & 17.4 & 100.0 \\
1986 & 47.3 & 24.4 & 5.5 & 5.4 & 17.4 & 100.0 \\
1987 & 45.7 & 25.1 & 5.6 & 5.8 & 17.8 & 100.0 \\
\hline
\end{tabular}

- Cilras originales más provisiones para transferencias de funcionamiento.

* Cifras Preliminares.

Fuento: Ministerio de Haciende. 
miento mismo de la burocracia gubemamental. ${ }^{3}$ Como puede verse, el impacto del conflicto armado sobre las finanzas públicas va más allá de un simple problema contable de ingresos-gastos. En realidad, su impacto mayor consiste en que su existencia imposibilita al gobiemo cumplir de manera satisfactoria sus funciones más elementales.

Efectivamente, y debido a las exigencias que le impone el conflicto, y ante la imposibilidad de obtener mayores ingresos, el gobierno se ha visto en la necesidad de reorientar los fondos disponibles afectando severamente a todos aquellos gaslos no relacionado con los esfuerzos de guerra. En los primeros afios de la crisis se recurrió a recortar los gastos de capital, los cuales desde entonces han sido financiados con recursos provenientes de la ayuda externa, especialmente de A.I.D. Luego, y ante las mayores demandas que impuso el conflicto, se procedió a recorlar los gaslos de funcionamiento los cuales a estas alturas ya no se pueden seguir reduciendo, sin afectar sensiblemente la ya deteriorada gestión técnico-administrativa del estado.

El hecho de que el aumento de los gaslos no haya lenido su contrapartida en el lado de los ingresos, ha posibilitado la aparición del déficit fiscal, el cual resulta difícil de manejar en el plazo, debido a la rigidez que presentan tanto los ingresost como los gastos. Esto significa que la reducción del déficit fiscal en El Salvador liene un límite, bo cual, liene implicaciones importantes en terminos de politica económica, ya que reduce suslancialmente sus margenes de acción.

¿Cómo asegurar la prestación de un nivel minimo de bienes y servicios que permitan el funcionamiento del gobierno, en un contexilo de una inflación relativamente alta y necesidades crecientes? ¿Cómo hacer frente al creciente deterioro de los servicios sociales? ¿Como satisfacer las demandas legítimas de los servidores públicos sin provocar mayores distorsiones en el resto del sistema económico? Estas son algunas de las preguntas que enfrenta la politica económica hoy en el área fiscal y que seguramente tendrá que enfrentar en el futuro, aún bajo el supuesto de que la guerra termine.

\section{II.1.2. La relación entre confllcto armado e Inflación}

Otra consecuencia directa del conficto es su impacto sobre la inflación. Contrariamente a lo que se argumenta, la presión de aquel sobre ésla no se realiza principalmente via déficil fiscal. ${ }^{5}$

A nuestro entender, la relación entre conflicto amado-inflación debe ser abordada desde otra perspectiva. De hecho, la influencia del conflicto sobre la inflación ocurre a través de otros mecanismos, siendo los más importantes su presión sobre la oferta, sobre la demanda y las cadenas especulalivas persistentes y permanentes que genera. 
Por el lado de la oferta, la existencia del conflicto ha provocado, por una parte, una reducción importante en la producción especialmente de granos básicos; y por otra, ha producido un aumento importante en los costos de las empresas industriales productoras de bienes-salario, todo lo cual se ha traducido en una presión permanente sobre el nivel de precios internos. Uno de los lactores que más presión ha puesto en los úllimos afios en los costos de las empresas ha sido el sabotaje permanente al sistema eléclrico del pals. Esla ha provocado que las empresas incurran en forma permanente en gaslos extraordinarios en plantas generadoras de energía eléctrica, gastos de combustibles, pérdida de producción por cortes de energía, en gastos en seguridad, los que a su vez han sido trasladados al precio del producto.

Por el lado de la demanda, el aumento y la desviación de los gastos del gobierno a aclividades improductivas (es decir, a los esfuerzos de guerra) ha sido un factor importanle que también ha pueslo presión sobre los precios. Asímismo, el fenomeno de la hipenurbanización, ocurrida en el país como produclo del confliclo es un tactor importante que ha contribuido al alza de los precios. Como es sabido, el conflicto ha provocado en El Salvador un desplazamiento poblacional de las áreas rurales hacia las principales ciudades del pais y de las ciudades pequekas a las más grandes. Esto, a la vez que ha contribuido a reducir el espacio economico, ha generado una luerle demanda sobre los principales bienes salarios, especialmente alimentación y vestuario, lo que a su vez ha provocado una presión sobe los precios de dichos bienes. En este sentido, no es casualidad que sea la zona oriental la que ha experimentado los mayores niveles de precios del pais, en los últimos anos.

Finalmente, los conlinuos procesos de desabastecimiento y especulación generada por acciones guerrilleras, y especialmenle por los paros al transporte terrestre son un elemenlo que juega permanentemente en el alza de los precios. Según estadisticas, el mes en que ocurre un paro, la inflación liene una elevación importante; eslo, debido a la escasez provocada por el abastecimiento de muchos productos -principalmente los que vienen de Guatemala- y a la enorme especulación que se genera una vez se liene conocimiento del mismo.

Así, los efectos directos e indirectos del contlicto sobre la inflación son multiples, y lo que es más importante permanentes. Dado que los elementos anteriores tienen carácler permanente, las presiones sobre el nivel de precios lambién son permanentes, por lo que las posibilidades de lograr avences significativos en su reducción son limitados. Dicho de otro modo, debido a la influencia permanente que pone el conflicto sobre el nivel de precios internos, ésta tiene un limile más allá del cual no se puede reducir. 
La consecuencia de esta siluación en lerminos de política económica son importantes. En primer lermino, esto significa que la postergación de los esfuerzos de reactivación a la espera de lograr niveles de estabilización significativamente menores a los actuales, es poco realista. En segundo lugar, y visto de otro modo, supone que el disefo de cualquier paquete de medidas tiene que tomar en cuenta sus electos sobre la inflación, ya que de otro modo ésta puede escapar facilmente de control. En este sentido, la inflacion se convierte en el elemento determinanle para el diseno de cualquier tipo de medidas, especialmenle, y como veremos más adelante, de aquellas tendientes a superar el desequilibrio del sector externo.

\section{II.1.3. La Influencla del conflleto amado en la produccion}

Las repercusiones del confliclo armado sobre la producción parecen más evidentes. Pese a que en los últimos anos se ha producido una relativa adecuación de los diferentes agentes económicos al marco general que impone el contlicto armado, ${ }^{6}$ las repercusiones de ésle sobre la evolución de la producción real son lodavía significativas. Especial importancia tiene la influencia del conflicto en la evolución de dos de los más imporlantes sectores de la economía salvadorena; el agropecuario y el industrial, los cuales representan alrededor del 42 por ciento del PIB. EI sector agropecuario se ve alectado por el conflicto de diversas maneras. Por un lado, debido al hecho de que la mayor parte de acciones bélicas se desarrollan en el área rural: lo cual, además de provocar el abandono de importantes zonas agrícolas, enlorpece permanentemente el desarrolo de las actividades de esa área. Por otra parte, las acciones de sabotaje directa a plantaciones o instalaciones agrícolas ha sido un factor que en los últimos meses ha provocado serios dahos y ha creado un marcado pesimismo en muchos agricullores. ${ }^{7}$

El otro sector que actualmemte está siendo seriamente afectado por las acciones bélicas es la industria. Ciertamente, las acciones de sabolaje emprendidas por la guerrilla contra el sistema eléctrico del pais, han ocasionado serios danos a la industria, la cual ha sufrido importantes pérdidas en terminos de producción perdida, $y$ ha incurrido en importantes costos adicionales lales como la adquisición de planlas generadoras de energía eléctrica, el gasto en combustibles los costos de paralización de labores, y los costos de seguridad. ${ }^{8}$ Las implicaciones de esta situación en lerminos de política económica son importantes. En primer lugar, la persistencia de acciones bélicas en el campo, imposibilita cumplir con los planes de producción que se disenan para el sector, e impiden avanzar en la ejecución de proyecto de inversión en el agro. En el caso de la induslria, y como ya se mencionó anteriormente, el aumento de las coslos se traduce en una presión permanente sobre los precios, y 
por lanto en una presión sobre el marco macroeconómico general.

\subsubsection{Otros efectos del confllcto armado}

Además de lodas las repercusiones anteriores, el conflicto armado tiene efectos importanles en otras áreas de la economía, y particularmente en la política económica y en la planificación económica.

Ciertamente, uno de los efectos colalerales que provoca la existencia misma del conflicto es la dificultad de efectuar una planilicación integral, y la imposibilidad de llevar adelante muchos de los planes, que se disefian, especialmente los regionales.

Como puede deducirse de lo expueslo hasta aqui, la influencia del conflicto armado en la evolución y el manejo de la política económica es decisiva. En realidad, y en lanto prolundiza y agudiza los problemas tradicionales y crea otros nuevos, distrae recursos que de otra manera podrían dedicarse a actividades productivas y sociales, genera inseguridad, imposibilita planificar y enlorpece y dificulta las diferentes acciones que se hacen para superar la crisis; el confliclo armado es el límite insuperable a todo esfuerzo verdadero de reactivación económica del pais.

Por otra parte, y en lanto su existencia impone límites a la reducción de los principales desequilibrios macroeconómicos, éste limila grandemente el márgen de acción de la politica económica.

Lo anterior, ha provocado que muchos de los objelivos y melas diseñadas por el gobierno no se haya cumplido a cabalidad, convirtiendose el conflicto de esta manera, en un obstaculo importante para la política económica y para la superación de la crisis.

\section{II.2. El limite que Impone a la política económica la talta de consenso}

Otro factor fundamental que en el caso de El Salvador se convierte en un límite al manejo de la política económica, es la falla de consenso de los diferentes sectores de la vida nacional (eslimulada en gran parte por el conlliclo). Como lodos sabernos, la politica económca de por sí es un condicionante primordial del consenso, razón por la cual algunos autores argumenlan que su eficacia debe medirse lambién en función de su contribución a la persuasión; es decir, en hacer que los comportamientos económicos se ajusten tendencialmente al proyecto que se trata de implementar. ${ }^{9}$

En el caso de El Salvador, y debido a los elevados niveles de polarización existentes, la política económica no sólo no ha sido capaz de cumplir con su función de persuasión, sino que incluso, se ha convertido muchas veces en un elemento más de confrontación. Esto es precisamente 
uno de los factores que explican el poco éxito alcanzado en terminos económicos en el periodo de crisis. Cierlamente, y en el marco general que impone la guerra y la crisis social, la política económica se ha convertido en un campo de lucha, en donde la discusión seria y objeliva sobre los problemas económicos y sociales del pais, ha sido desplazada por confrontaciones ideológicas, las más de las veces antagónicas.

En este contexto, por muy bien disenada técnicamente que estén las políticas, éstas generalmente no alcanzan los objetivos y melas para las que fueron pensadas, $y$ en algunos casos, hasta arrojan resultados contrarios. Esto se debe a que los diferentes agentes económicos y sociales no solo no responden de acuerdo a los comportamientos esperados, sino que muchas veces actuan en sentido contrario. En este caso, las posibilidades de la política económica se reducen suslancialmente.

Un elemento adicional que a partir de 1984 ha contribuido a hacer de la política económica un elemento más de confrontación ha sido la falta de alinidad política de diversos sectores con el actual gobierno, especialmente de algunos de la empresa privada. Esta siluación, se ha Iraducido en la práctica en una confrontación permanente, lo cual a la vez que ha reducido las posibilidades de éxito y los márgenes de acción de la política económica, ha convertido a ésta en un campo privilegiado de confromlación entre ambos sectores.

\section{II.3. El sector externo como el princlpal limite al crecimiento econo- mico y el principal punto critlco de la politica económica}

Al igual que el resto de las economias certroamericanas, la salvadorefia se caracteriza por su extremada dependencia lecnológica. Los bienes intermedios (materia prima, productos semi-procesados, petróleo, fertilizanles, elc.) junto a los bienes de capital, representaron en promedio más del 70 por ciento de las importaciones totales para el periodo 1980-1987. (ver cuadro No. 3)

\section{Cuadro No. 3}

EI Salvador: Clasillcaclón económica de las Importaclones (C.I.F.) (Porcentaje distribución por categorias)

\begin{tabular}{|l|r|r|r|r|r|r|r|r|}
\hline & 1980 & 1981 & 1982 & 1983 & 1984 & 1985 & $\begin{array}{c}\text { Programa. BCR } \\
1986\end{array}$ & 1987 \\
\hline Total de importa- & & & & & & & & \\
ciones (CIF) & 100.0 & 100.0 & 100.0 & 100.0 & 100.0 & 100.0 & 100.0 & 100.0 \\
Bienes de consumo & 31.9 & 30.7 & 30.5 & 29.4 & 28.3 & 26.9 & 24.4 & 24.3 \\
Bienes de intermedios & 56.6 & 57.8 & 59.2 & 59.7 & 58.2 & 56.7 & 57.7 & 52.1 \\
Bienes de capital & 11.7 & 11.6 & 11.6 & 11.7 & 13.5 & 16.4 & 17.9 & 23.5 \\
\hline
\end{tabular}

Fuente: BCR. 
Por esta razón, las posibilidades de lograr un crecimiento económico depende de la capacidad que tiene el pais de importar. En realidad y tal como puede observarse en el Cuadro No. 4 cada vez que la economia crece, necesita de un nivel de importaciones mayor que el crecimiento del producto, lo cual como veremos en seguida, se convierte en una seria limitación a la balanza de pagos, y al crecimiento económico.

Cuadro No. 4

El Salvador: Crecimlento promedlo por perlodos de las Importaclones de blenes y del PIB 1962-1987

(En porcenlajes)

\begin{tabular}{|l|c|c|}
\hline Periodo & $\begin{array}{c}\text { Tasa de crecimiento } \\
\text { real del PIB }\end{array}$ & $\begin{array}{c}\text { Tasa de crecimiento } \\
\text { de las importaciones }\end{array}$ \\
\hline $1962-1967$ & 6.3 & 12.4 \\
$1967-1972$ & 4.0 & 4.3 \\
$1972-1977$ & 5.4 & 27.8 \\
$1977-1982$ & -3.7 & -1.6 \\
$1982-1987$ & 1.6 & 16.3 \\
\hline
\end{tabular}

Fuente: Elaborado en base a dalos del Banco Central de Reserva.

Dado el grado de relativa rigidez que presentan las exportaciones salvadoreñas, así como su drástica reducción ocurrida en el perlodo de crisis, lo cual se ha traducido en délicil de la cuenta comercial, el sector Externo se convierle sin duda, en el principal límite a cualquier esfuerzo encaminado a lograr una verdadera reactivación de la economía salvadoreña.

Por la imporlancia que este punlo tiene en terminos de política económica, es necesario estudiar que es lo que ha pasado en el sector externo durante los últimos anos.

Sin duda, el indicador más evidente del grave problema que presenta la economia salvadorefia en su sector externo, es el déficit creciente registrado en la cuenta comercial de la balanza de pagos (Ver cuadro No. 5).

Dicha cuenta, que en 1979 mostró un superávit de US $\$ 92.2$ millones, posteriormente se fue deteriorando hasla registrar un déficit record de. US \$ 402.6 millones en 1987.

Como puede observarse, dicho comportamiento se explica básicamenle por la evolución mostrada por las exportaciones, las cuales a partir de 1980 experimentan una caida pronunciada, a tal grado que su valor 
Cuadro No. 5

El Salvador: Balanza comerclal, periodo 1979-1987

(En millones de dólares)

\begin{tabular}{|c|c|c|c|c|c|c|c|c|c|}
\hline & 1979 & 1980 & 1991 & 1882 & 1983 & 1994 & 1985 & $\begin{array}{c}(1) \\
1986\end{array}$ & $\begin{array}{c}\text { (2) } \\
1987\end{array}$ \\
\hline \multicolumn{10}{|l|}{ I. Exportaciones de bienes } \\
\hline (FOB) & 1131.3 & 1073.6 & 796.7 & 699.5 & 757.9 & 725.9 & 695.1 & 754.9 & $\mathbf{5 7 2 . 7}$ \\
\hline - Calé & 675.2 & 615.2 & 452.6 & 402.6 & 438.2 & 443.9 & 457.9 & 539.1 & 347.3 \\
\hline - Algodón & 84.6 & 84.8 & 53.6 & 45.2 & 55.4 & 9.1 & 29.0 & 4.5 & 2.3 \\
\hline - Azúcar & 26.8 & 13.4 & 14.8 & 15.9 & 40.1 & 25.9 & 23.2 & 25.3 & 12.1 \\
\hline - Camarón & 12.3 & 12.8 & 16.0 & 18.2 & 11.7 & 20.2 & 9.8 & 17.0 & 20.5 \\
\hline $\begin{array}{l}\text { - No Iradicionales al MCCA } \\
\text { - No tradicionales al resto }\end{array}$ & 266.6 & 295.8 & 206.5 & 174.2 & 164.9 & 157.2 & 95.7 & 91.0 & 111.1 \\
\hline del mundo & 65.8 & 51.8 & 53.2 & 43.4 & 47.6 & 69.6 & 79.5 & 78.0 & 79.4 \\
\hline \multicolumn{10}{|l|}{ II. Imporlaciones de bienes } \\
\hline (CIF) & 1039.1 & 961.7 & 984.6 & 056.8 & 892.8 & 977.5 & 961.4 & 934.9 & 975.3 \\
\hline III. Balanza comercial (I - II) & 92.2 & 111.9 & -187.9 & -157.3 & 134.9 & -251.6 & -266.3 & -180.0 & -402.6 \\
\hline
\end{tabular}

(1) Cilras preliminares ejusıadas con datos de INCAFE, COPAL $\theta$ INAZUCAR.

(2) Cilras proyectedas.

en 1987 lue la mitad de lo obtenido en 1979. Las importaciones por su parte, como produclo del aumento en su valor unitario y del elevado componente importado con el que opera el aparato productivo salvadorefio, han permanecido en el periodo de crisis en niveles relativamente allos, disminuyendo en una proporción menor al de las exportaciones, 10 cual también ha contribuido a acentuar la brecha de la cuenta comercial.

Hasla hoy, dicha siluación en el sector externo ha podido ser mantenida gracias a la ayuda norleamericana y a los ingresos en concepto de remesas familiares. Esto, como se ha sefialado muchas veces, ha postergado la necesidad de realizar ajustes, el cual, es sin duda inescapable. 10

La gravedad del desequilibrio externo, y la dificultad de reducirlo signlicativamenle en el corto plazo, convierte al sector externo en el principal límite a cualquier esfuerzo de reactivación económica. A su vez, y como veremos en seguida, es lambién el principal "punto critico" en cuanlo al diseño y manejo de la política económica debido a que algunas de las principales políticas que generalmene se adoptan para reducirlo, aclúan muchas veces en sentido contrario a los esfuerzos de estabilización.

Como veíamos en la parte referente a las repercusiones del confliclo sobre la inflación, ésta tiene un límite que imposibilita reducirla significativamente de los niveles acluales. En este contexto, la politica eco- 
nómica tiene que manejarse prudentemente, de manera de mantenerla en tales límites.

Es decir, la política económica liene que buscar un mínimo de estabilización, lo cual, obviamente requiere un manejo cuidadoso de ésta, ya que de lo conlrario la inflación rapidamente puede escapar de conlrol.

Es precisamente esta necesidad de mantener un nivel minimo de eslabilización, el principal elemento condicionanle a cualquier programa de ajuste tendiente a superar el desequilibrio del sextor externo, y esto es así debido a que si bien la s políticas de ajuste -orienladas a reducir el desequilibrio externo-, y las políticas de estabilización, -dirigidas a disminuir la inflación-, son compatibles entre si en algunos aspectos, como es el caso de la necesidad de controlar la expansión de la demanda agregada; también puede presentar contradicciones en otros, como por ejemplo, cuando para reducir el déficit externo se opla por medidas como la devaluación, haciendo de esta manera más dilícil $-\mathrm{y}$ a veces hasta imposible- la estabilización debido a su efecto alcisla sobre los costos.

Este es precisamente el caso salvadorefio. Tal y como algunos esludios han sefialado'11, uno de los principales elementos que explican la inflación en los últimos años, es el proceso de devaluación que se inició a partir de mediados de 1982 y que culminó con la unilicación del lipo de cambio en enero de 1986.

De hecho, este proceso puso presión sobre los precios de dos maneras. Por una parte, debido a su impacto en los coslos de producción y por otra, como consecuencia del relorzamiento de las expectativas inflacionarias, las cuales, en el caso de El Salvador, son uno de los principales factores propagadores de la inflación.

Debido a lo anterior, y en términos de manejo de la polílica económica, la necesidad de mantener niveles minimos de estabilización es el principal elemento a considerar para el diseño de ajuste requerido. De hecho, cualquier paquete de medidas de ajuste tiene que evaluarse en función de su impacto sobre el nivel de precios internos, ya que solo asi pueden visualizarse los limites del ajuste.

Las implicaciones que ésto liene para la política económica son evidentes: la posibilidad de avanzar en un proceso de reactivación económica supone la superación del desequilibrio externo, no obstante, y debido a la estructura presentada por el sector externo, así como el limite al ajusle que impone la necesidad de asegurar un minimo de estabilización, los márgenes de la política en este campo son realmente. estrechos. Dicho en olras palabras, las posibilidades de lograr crecimienlo y estabilidad de precios dependen, en el caso de El Salvador, de la forma en que se enirente el desequilibrio externo. Dado que las medidas tradicionales que generalmente se utilizan para su superación en- 
tran en contradicción con los objetivos de estabilizacion, el desequilibrio externo, se convierte en el principal punto crítico en el manejo y el diseno de la polílica económica de EI Salvador.

\section{II.4. El límlie a la politlca economlca derlvado de la alta dependencla de la ayuda externa}

Para nadie es un secreto la alia dependencia de la economía salvadoreña de la ayuda externa, y particularmente de la proporcionada por el gobierno de los Estados Unidos a Iravés de la Agencia para el desarrollo Internacional (AID). Como puede verse en el cuadro No. 6 dicha asistencia alcanzó los US $\$$ 1.762.0 millones en el período 1983-1987.

\section{Cuadro No. 6}

Prestamos y donaclones de la AID (En millones de dólares)

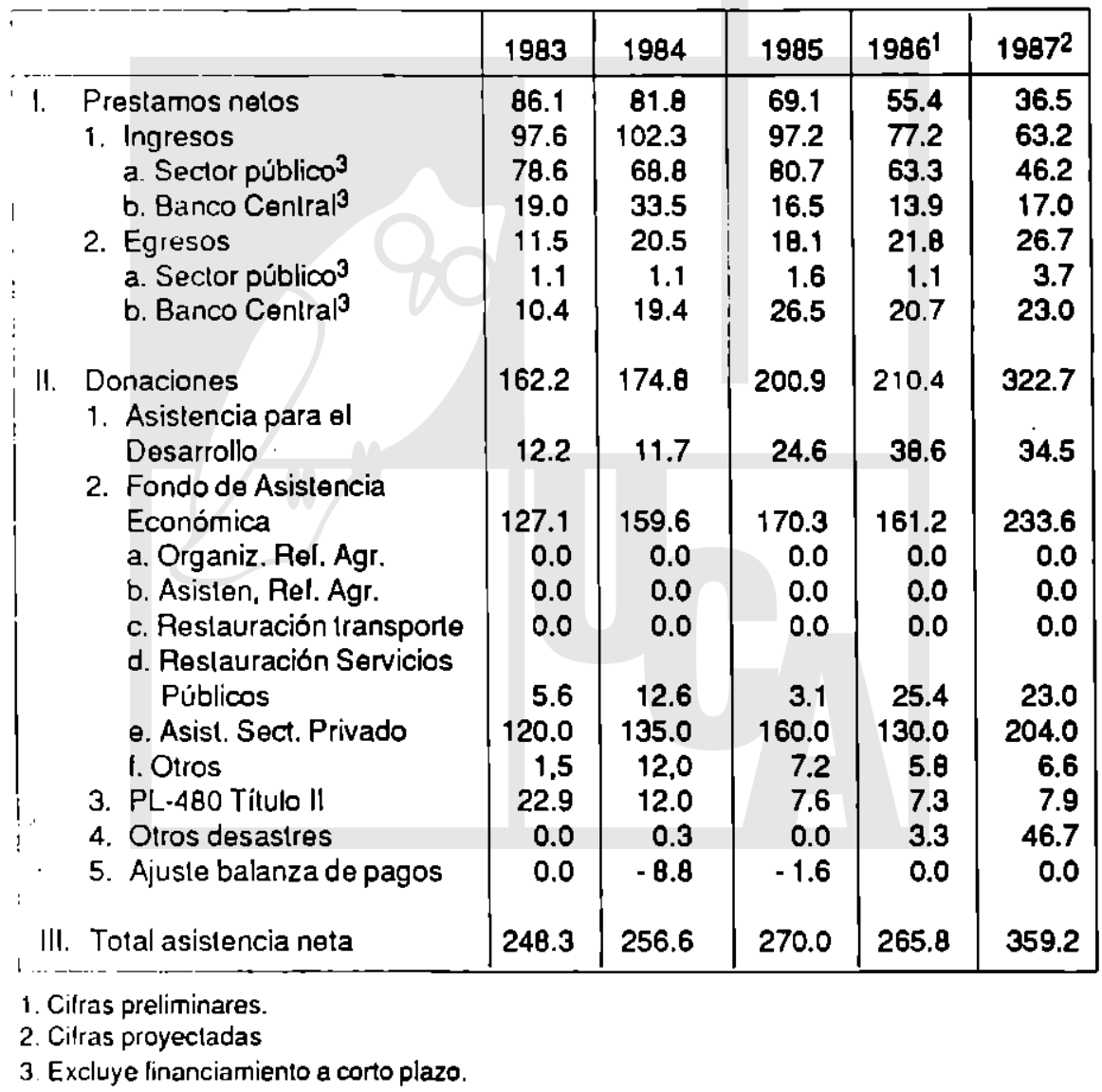


La ayuda externa ha jugado en el pais un papel decisivo. En tanto ha permitido contar con las divisas suficientes para efecluar las importaciones que requiere el aparato productivo local para su funcionamienlo, no solo ha evitado el colapso de la economia salvadorefia, sino que también ha posibililado la postergación del ajuste externo, el cual de otra manera hubiera sido inevitable. Por otra parte, es esta ayuda la que ha permitido al gobierno realizar algún tipo de inversión tísica, y darle un mantenimiento mínimo a la esiructura económica social.

En este conlexio, la ayuda extema en El Salvador ha dejado de ser un elemento complementario al esluerzo interno, y se ha convertido en parte constiluyente y constitutiva del funcionamiento de la economía salvadorena.

En realidad, la importancia de esta ayuda es tal, que sin ella la crisis económica, y particularmente los desequilibrios inlernos y externos estarian lotalmente luera de control. De hecho, la dependencia de la ayuda externa alcanza niveles tan allos, que una reducción sustancial de la misma, puede provocar cambios fundamentales en la estruclura económica y en el modelo político. ${ }^{12}$

Esta dependencia tan alta de la ayuda externa tiene serias implicaciones en el manejo y el diseno de la polílica económica del país. En primer término, reduce considerablemente sus márgenes de acción, ya que dicha ayuda generalmente viene acompanada de toda una serie de condicionalidades, las cuales no necesariamente coinciden con las prioridades del gobierno y las necesidades del país.

En cuanto al diseño de la política económica, y concretamente el eslablecimiento y logro de las melas macroeconómicas, la dependencia de la ayuda se hace evidente. Cientamente, las metas macroeconómicas que se tijan para cada ano, se establecen en función del monlo de la ayuda que se espera recibir, y la mayorla de las acciones que se programan dependen de la agilidad y fluidez con que ésle ingrese.

Dicho de otro modo, todo el Programa Económico que se elabora para cada ano depende de el monto y de la rapidez con que ingrese la ayuda. Si por cualquier motivo ésle no ingresa según la candelarización eslablecida, las posibilidades de alcanzar las metas propuestas se reducen considerablemente.

II.5. El límite a la politica económica que Impone el poco conoclmlento de la realldad que se qulere transformar

Como producto de la misma crisis económica y de las políticas y acciones implementadas, la eslructura económica salvadorefia ha experimentado durante los últimos años una serie de transformaciones que 
sin duda han provocado cambios en su funcionamiento y en su composición.

Desgraciadamente, y debido a la conjugación de una serie de factores, el conocimiento que se tiene de dichas Iranstormaciones es muy limitado, lo cual imposibilila disefiar las medidas acordes a la naturaleza de los cambios ocurridos. En realidad, y debido a esta limitación, el diseño de polílicas específicas para sectores especílicos resulla una tarea sumamente dificil y compleja. De hecho, y debido al poco conocimiento de la realidad económica salvadoreña, muchas políticas se disefian en base a "percepciones" e "intuiciones," lo cual, obviamente, limila sus posibilidades de éxito.

Por otra parte, y debido a esta limitación, la política económica que se disef́a se queda generalmente a un nivel demasiado agregado, reduciendo de esta manera su margen de acción, y provocando muchas veces resultados no buscados.

Por si esto fuera poco, y debido, a las especílicidades que presenla la economía, los enfoques teóricos tradicionales, asi como las recomendaciones de polílica que de ellos se derivan, son incapaces de dar respuesta a los grandes problemas que presenta el pais.

La conjugación de estos dos elementos; es decir, el poco conocimiento de la realidad y la incapacidad de la teoría para dar cuenta de esa realidad, hacen que hacer política económica en El Salvador sea una tarea realmente dificil, y que las posibilidades de lograr avances significalivos de la superación de la crisis se reduzcan significativamenle.

\section{Algunas reflexlones finales}

Los límites, dilemas y contradicciones que enfrenta la política económica en El Salvador, reflejan de una forma clara la complejidad y la gravedad de la crisis que afecla el pais, y arrojan dos conclusiones importantes. En primer lugar, el análisis de las páginas precedentes sugiere que dada la gravedad y complejidad de la crisis económica del país, su superación tiene que ser necesariamente gradual. En segundo término, y es lo más importanle, dada la naturaleza de los diversos factores que intervienen en la configuración de la crisis, y que limilan el márgen de acción de la política económica, su superación requiere necesariamente la participación de todas las fuerzas sociales, políticas y económicas del país. Y esto es asl no sólo porque la viabilidad de cualquier programa o estrategia así lo exige, sino porque la remoción de muchos de los obstáculos que actualmente impiden avanzar en la solución de la crisis son de orden político, y pasan necesariamente por la discusión de que es lo que se quiera para un país en el futuro. 
En este sentido, las posibilidades de superar la crisis económica del país estan en función directa de los avances que se logren en el ámbito polílico, y concretamente en la solución del conflicto armado, y en los esluerzos encaminados a lograr un mínimo de consenso alrededor de un proyecto económico.

Sólo dentro de este contexto la polílica económica dejará de ser un elemento de confrontación y un insirumento de simple "administración" de la actual crisis económica, para convertirse en lo que verdaderamente debe ser, es decir, un instrumento de cambio y transformación y un elemenlo que contribuya a lograr un mayor nivel de consenso nacional.

Sólo asi la política económica estará en capacidad de superar los límiles, dilemas y contradicciones que enfrenta actualmente y que por el momento parecen insalvables.

\section{NOTAS:}

- Ponencia presentada en el Encuentro de opciones de política econórnica para EI Salvador, patrocinado por la Maestrla en politica económica de la Universidad Nacional de Costa Rica, en mayo de 1988.

1. Tal y como se ha señalado, esle conceplo de polftica económica diliere del corriente, limitado el manejo por parte del Estado de instrumentos considerados "económicos" (precio, tasa de cambio e interés, moneda, etc.). Ver Corragio, J.L. "Economla y póltica en la transición. Reflexiones sobre la Revolución Sandinista" en la transición dilicil. Siglo Veintiuno, Editores, 1986, pág. 259.

2. Esto no significa que la crisis económica sea producto exclusivo de la guerra, ni que en el marco general que ésta impone no se puede realizar algún tipo de "administración" de la misma.

3. Dicha situación se vió agravada por el terremolo del 10 de octubre de 1986, que alectó seriamente a la administración pública. Actualmente, existen problemas de abastecimiento de material básico. La falta de papelerla, material de oficina, etc., es ya la regla en muchas dependencias gubemamentales.

4. Por el lado de los ingresos, la posibilidad de aumenlarlos sustancialmenle en el corto plazo son muy limitadas, a menos que se recurra al aumento de los impuestos indirectos, lo cual es improbable por las implicaciones pollticas que supone. La reciente relorma tributaria (que alectó a los impuestos directos) cierra en el corto plazo las posibilidades de actuar en esa vla, pese a que se puede avanzar mucho en este campo mediante una reforma administrativa, y sunque ya se están dando algunos pasos al respecto, sus resultados se reflejarán más bien en el mediano plazo.

5. Contrariamente a lo que se supone, el délicit fiscal no parece ser el elemento explicativo principal de la inflación en El Salvador. En realidad, es la inflación la que en los últimos años le ha puesto una enorme presión al déficit liscal, ya que ha significado para el gobierno mayores erogaciones para adquirir la misma cantidad de bienes y servicios. Para un enloque alternativo de la inflación, ver: Rivera $\mathrm{C}$. Roberto. La inflación en El Salvador. Revista Económico-Social, año 1, No. 1 Enero-febrero/8.

6. Este hecho ha sido señalado ya por instituciones como CEPAL y el BID. y es lo que explica en parte el dinamismo mostrado por la inversión privada, la cual creció en el perlodo $1984-1987$ a una tasa promedio anual del $15.0 \%$.

7. A partir del último trimestre de 1997, la guerrilla comenzó a desarrollar una nueva estrategia de sabotaje a la economia, consistente en la destrucción Ilsica de plan- 
taciones. instalaciones, haciendas ganaderas, etc. A estas alturas muchas instalaciones agricolas han sido destruidas, centenares de cabezas de ganado encrificadas y muches plantaciones egricolas (especialmente de cal's) hen sido quamadas.

8. Sin duda, el sabotaje al sistema eléctrico realzado por la guerrilla es una de las acciones que más afecta la economla del pals. Además de los electos en la industria, los daños en el comercio, los servicios y el transporte son también significativos.

9. Ver Coraggio. Op. cit. pág. 260.

10. Ver Pleitez W. y Segovia A. Los electos de una devaluación en la Economla Sa!vadoreña en el marco de un proceso de Estabilización y Reactivación. Revista Presencia, eno, No. 2, 1988.

11. Ver Rivera, R. op. cit.

12. Ver Envio, año 5, No. 55-56 enerollebrero 1986. 